\title{
Recommandations pour la pratique clinique Cancer du rectum
}

\section{Conclusion}

\author{
Y. Panis \\ (C) Lavoisier SAS 2016
}

La relecture (studieuse) de ces 7 questions amène à la réflexion suivante : le cancer du rectum est un sujet à la fois :

- très simple sous plusieurs aspects : peu d'examens à demander en pré-thérapeutique, exérèse totale du mésorectum (TME) maintenant bien standardisée, indications très claires du traitement local des petits cancers du rectum,

- mais aussi parfois en complète évolution : notamment pour le concept de préservation d'organe (est-ce bien raisonnable de ne pas faire de TME chez certains patients ? Comment diagnostiquer une réponse vraiment complète?), ou encore par exemple pour celui du « prétraitement » par chimiothérapie avant une éventuelle radiochimiothérapie néoadjuvante chez des patients sélectionnés,

- sans parler de sujets où il est vraiment difficile en 2016 d'avoir une idée bien arrêtée : quid du robot qui coûte cher, mais qui, à n'en pas douter, va se développer dans les années à venir avec de nouveaux modèles ? quid de la
TME par en bas ? Réel progrès ou simple mode passagère?

En conclusion, on se dit que finalement, il faudra certainement faire la troisième version des RPC...dans 10 ans voire moins !

On peut déjà remercier l'ensemble des responsables des questions et les différents groupes d'experts qui ont participé à la rédaction, mais aussi au groupe de lecture, pour l'énorme travail qui a été réalisé, car, comme d'habitude, votre serviteur a simplement initié ce projet de mise à jour, puis beaucoup harcelé les responsables de questions, et enfin un peu relu les textes intermédaires et définitifs... mais il a clairement beaucoup moins travaillé que Jérémie Lefevre, Valérie Bridoux, Cécile de Chaisemartin, Sylvain Kirzin, Zaher Lakkis, Léon Maggiori, Antoine Brouquet, Gilles Manceau, et leurs différents groupes de travail.

Merci donc d'abord à eux pour ce très beau travail.

Y. Panis $(\bowtie)$

Service de Chirurgie Colorectale,

Pôle des Maladies de l'Appareil Digestif (PMAD),

Hôpital Beaujon, 100, bd du Gal Leclerc,

92118 Clichy cedex, France

e-mail : yves.panis@bjn.aphp.fr 